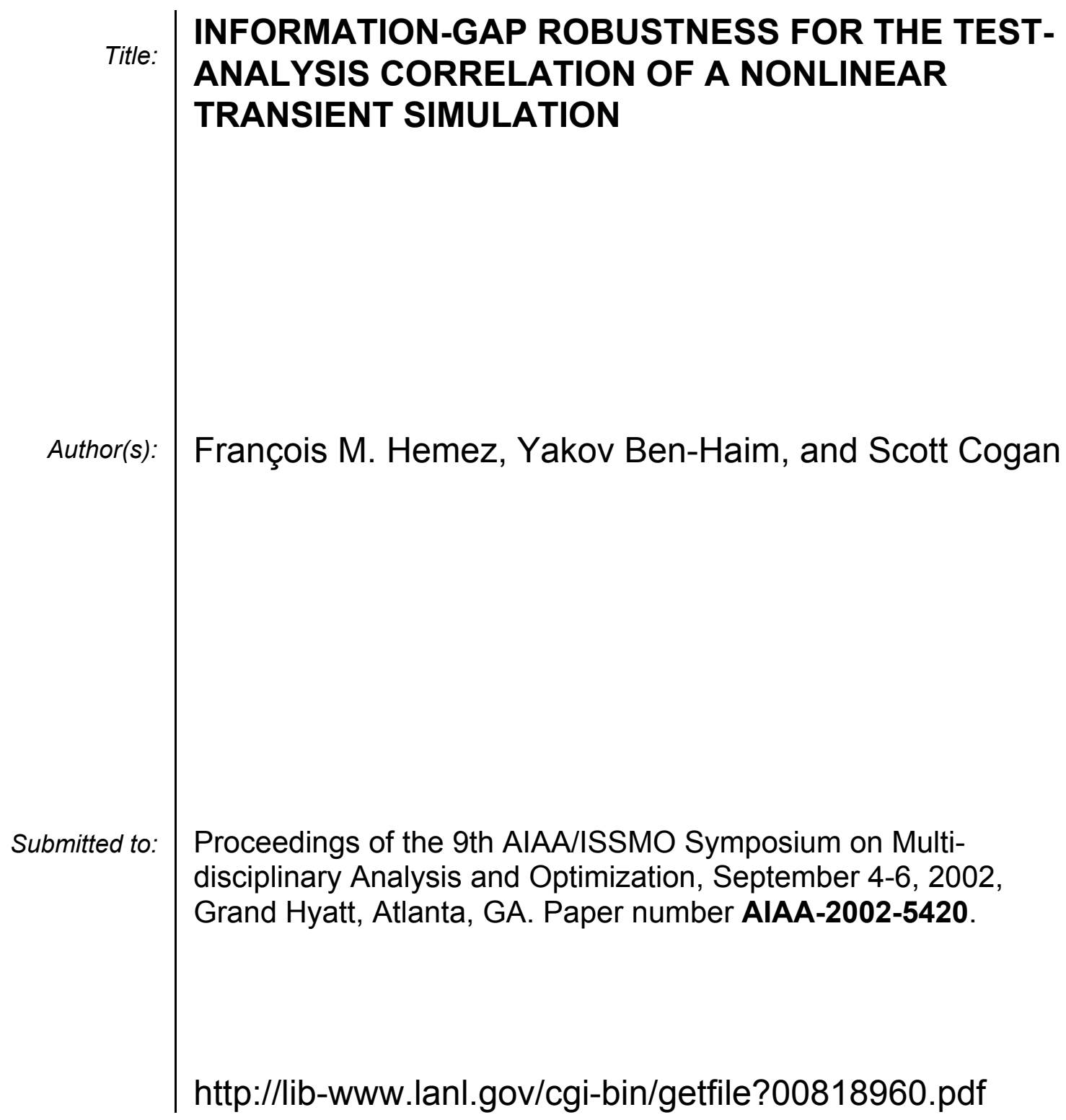




\title{
INFORMATION-GAP ROBUSTNESS FOR THE TEST-ANALYSIS CORRELATION OF A NONLINEAR TRANSIENT SIMULATION
}

\author{
François M. Hemez ${ }^{*}$ \\ Engineering Sciences and Applications, ESA-WR \\ Los Alamos National Laboratory \\ Mail Stop P946, Los Alamos, New Mexico, U.S.A.
}

\author{
Yakov Ben-Haim \\ Faculty of Mechanical Engineering \\ Technion-Israel Institute of Technology \\ Haifa 32000, Israel
}

\author{
Scott Cogan \\ Laboratoire de Mécanique Appliquée Raymond Chaléat \\ Université de Franche-Comté \\ 24, rue de l'Epitaphe, 25030 Besançon, France
}

\begin{abstract}
An alternative to the theory of probability is applied to the problem of assessing the robustness of test-analysis correlation to parametric sources of uncertainty. The analysis technique is based on the theory of information-gap, which models the clustering of uncertain events in families of nested sets instead of assuming a probability structure. The system investigated is the propagation of a transient impact through a layer of hyper-elastic material. The two sources of non-linearity are the softening of the constitutive law implemented to model the hyper-elastic material and contact dynamics at the interface between metallic and crushable materials. The robustness of test-analysis correlation to sources of parametric variability is first studied to identify the parameters of the model that significantly influence the agreement between measurements and predictions. Calibration under non-probabilistic uncertainty is then illustrated. Finally, two information-gap models of uncertainty are embedded to represent uncertainty not only in the knowledge of the model's parameters but also in the form of the model itself. Although computationally expensive, it is demonstrated that the information-gap reasoning can greatly enhance our understanding of a moderately complex system when the theory of probability cannot be applied due to insufficient information.
\end{abstract}

\section{INTRODUCTION}

One central difficulty in identifying the form of a numerical model and calibrating the values of its parameters is that the identification and calibration results can be ambiguous. Alternative combinations of model forms and parameter values can yield essentially equally good reproduction of test data. This ambiguity is sometimes analyzed in terms of analytical or numerical ill-conditioning or instability. This ambiguity is further exacerbated by the fact that not only decision variables are involved, but numerous other unknown or uncertain variables fluctuate beyond the control of the experimenter, and sometimes even without the experimenter's awareness of their presence or relevance. In short, ambiguity can occur whenever "variables"-meaning input parameters, models or conceptual forms - can interact to reproduce the data in more than one way.

Uncertainty plays a central role in calibration and ambiguity of identification. Clearly, conceptual ambiguity and numerical ambiguity are the result of imprecision or lack of information, both of which also result from uncertainty. Epistemic uncertainty occurs

\footnotetext{
*Technical staff member, "Validation Methods" team, hemez@lanl.gov, AIAA member.

† Professor, Yitzhak Moda'i Chair in Technology and Economics, yakov@ techunix.technion.ac.il.

Research Associate, French National Center of Scientific Research, scott.cogan@ univ-fcomte.fr.
}

Copyright $\odot 2002$ by F.M. Hemez (Los Alamos National Laboratory), Y. Ben-Haim (Technion Israel Institute of Technology) and S. Cogan (Université de Franche-Comté). Published by the American Institute of Aeronautics and Astronautics, Inc., with permission. 
in modeling activities when the laws that govern the evolution of a system are not known with absolute certainty. Parametric uncertainty originates from the relative imprecision with which parameters of a model are measured or identified. Uncertainty, rather than being an accident of the scientific method, is rather its very nature. (Adapted from a quote of Andrea Saltelli ${ }^{1}$.)

This paper addresses the problem of refining a numerical model to make it reproduce the test data to a given level of accuracy. To achieve this objective, the correct model form and parameter values must be inferred from the test data. In light of the above discussion, it implies that uncertainty must be accounted for and that the robustness of the identification to uncertainty must be established.

In structural dynamics where the preferred analysis method is the finite element method, the model calibration problem has been studied extensively. ${ }^{2}$ The work presented in this paper departs from the state-of-the-art in finite element model updating in two main ways. First, non-linear finite element models are developed to represent fast, transient events. Some of the materials involved exhibit a softening behavior and they cannot be represented with a linear constitutive relation. In addition, the dynamical phenomenon of interest is the highfrequency wave propagation. It occurs in less than one millisecond and cannot be represented using a truncated basis of low-frequency mode shapes.

Second, it is assumed that uncertainty cannot be represented through the conventional theory of probability. Probability theory is often taken for granted even though it can only be rigorously justified in cases when enough observations are available to estimate converged statistics. For example, assume that $X$ is a random variable with realizations $-0.19,+0.73$, $0.59,+2.18$ and -0.14 . If enough samples are available, statistics such as the mean and standard deviation can be computed. If more samples become available, it might even be possible to estimate the probability density function. However, if only the five samples of the previous sequence are available, all that can be said without adding more information that is really available, is that $X$ belongs to an interval of unknown size which is centered somewhere in $[-0.59 ;+2.18]$. Not being able to rely on probabilities implies that uncertainty propagation cannot take advantage of efficient sampling techniques. Therefore, the computational cost of the method proposed for nonprobabilistic uncertainty propagation should not come as a surprise.

Accepted for unlimited, public release on June 11, 2002.

American Institute of Aeronautics and Astronautics.

\section{UNCERTAINTY AND ITS EFFECT ON COMMON MODELING ACTIVITIES}

We start by discussing the effect of uncertainty on common modeling activities such as test-analysis correlation and model calibration. The discussion also serves the purpose of introducing definitions and notations used throughout this work.

\subsection{Representation of Model and Uncertainty}

The numerical model we seek to develop using the finite element method is simply denoted:

$$
y=M(q)
$$

The model provides a non-linear mapping between $y$, the output features (such as mean stress, peak acceleration, fundamental resonant frequency) and $q$, the model's input parameters (such as the constitutive model, shell thickness, friction coefficient). The selection of $q$ entails both the identification of the model's parameter values as well as the choice between conceptually distinct classes of models. A model specified by $q$ is validated when it can be asserted with confidence that $q$ accurately represents the physical properties of the system throughout the design domain.

In addition to the decision variables $q$, we deal with uncertainty variables $u$ represented by an information-gap model (IGM) denoted by $U\left(u_{0} ; a\right)$ :

$$
y=M(q ; u), \quad u \in U\left(u_{0} ; a\right)
$$

The unknown $u$ may represent a damping mechanism that we are not aware of, a coefficient of strain-rate dependency, a non-linear stiffness parameter, etc. We may have information about the uncertainty variables $u$, however this information may be quite fragmentary. We may not even know the identity of some of these uncertain variables. Or, we may be unsure whether a given variable should be categorized as decision variable $q$ or uncertainty variable $u$. This would translate the fact that the actual sources of uncertainty and their influence on the performance indicators $y$ are somewhat unknown.

\subsection{Immunity to Uncertainty}

The conventional paradigm for test-analysis correlation states that the decision variables $q$ can be calibrated to provide a model that reproduces the experimental data with high fidelity. Due to the ambiguity of identification, different models might be 
calibrated equally well. One model may be better than the others but we cannot know which.

Furthermore, even if a single choice of the decision variables $q$ reproduces the data with higher fidelity than all competing models, we still cannot have confidence that this maximally calibrated model is physically valid throughout the design domain. The reason is that the uncertainty variables $u$ interact with the decision variables $q$ and thereby preserve the potential for identification ambiguity. The quality of calibration may be due not to the physical validity of $q$, but to the coordination of $q$ with $u$. The highly calibrated variables $q$ may be an artifact of the attendant variables $u$.

However, when a calibrated model is obtained and this model is highly insensitive to variations of the uncertain variables, then the validity of the model can be established with confidence throughout the design domain. By establishing the immunity of the decision variables $q$ to the uncertain variables $u$, we ameliorate the interaction between the latter and the former and thereby reduce the ambiguity of the identification. This "factoring out" of the uncertain variables strengthens the confidence in the validity of the model throughout the design domain.

\section{INFORMATION-GAP MODELING OF UNCERTAINTY}

How to factor out the uncertainty variables without relying on the theory of probability is the focus of this Section. The information-gap models of uncertainty are briefly introduced. A formulation is then proposed to investigate the robustness of testanalysis correlation to sources of uncertainty.

\section{$\underline{\text { 3.1 Test-analysis Correlation }}$}

As explained previously, a numerical model is calibrated if it accurately reproduces the test data. The discrepancy between test data $y^{\text {Test }}$ and model predictions $y$ can be assessed with different metrics denoted by $R(q ; u)$. For instance, a mean-squared error metric may be defined:

$$
R(q ; u)=\sum_{k=1 \ldots N}\left(y_{k}^{\text {Test }}-y_{k}\right)^{2}
$$

Note that predictions $y$ of the model depend upon the decision variables $q$ as well as upon the uncertainty variables $u$. Whatever measure of discrepancy is employed, $R_{C}$ denotes the greatest level of infidelity that is acceptable. That is, a model is calibrated if:

$$
R(q ; u) \leq R_{C}
$$

A model is "good enough" or "acceptable" if it satisfies equation (3.2). In other words, $R_{C}$ specifies the level of satisficing for the model calibration. While the fidelity function $R(q ; u)$ depends upon the uncertain or unknown quantities $u$, it will turn out that this is not an impediment to the analysis. On the contrary, it is the prime mover of the info-gap robustness analysis to be discussed below. The uncertainty of variables $u$ is modeled by an IGM $U\left(u_{0} ; a\right)$ which is a family of nested sets parameterized by a coefficient $a>0 .^{3}$

\section{$\underline{\text { 3.2 Information-gap Modeling }}$}

Before proceeding with a robustness analysis, it is useful to briefly explain how the information-gap models of uncertainty are constructed. An IGM is simply a collection of sets of uncertain events. The "size" of these sets is controlled by the horizon-ofuncertainty parameter $a$. The sets, denoted $U\left(u_{0} ; a\right)$, are nested so that $a<a$ ' means that $U\left(u_{0} ; a\right)$ is included in $U\left(u_{0} ; a^{\prime}\right)$. In other words, the range of uncertain events increases as the uncertainty parameter $a$ increases. For example, describing the random variable $X$ of Section 1 with a convex IGM could consist of establishing the domain within which $X$ varies around a nominal value denoted by $X_{o}$ :

$$
U\left(X_{o} ; a\right)=\left\{X|| X-X_{o} \mid \leq a\right\}, \quad a \geq 0
$$

Equation (3.3) clearly shows that the potential number of values that variable $X$ can assume increases with the uncertainty parameter $a$. Other examples of info-gap models of uncertainty are provided in Sections 6, 7 and 8 and Reference ${ }^{3}$. The important point is that no probability structure is assumed. Instead, information-gap hypothesizes the structure of the uncertainty space and dictates how uncertain events cluster around one another. It is important to realize that the IGM assumption is much less restrictive than postulating the frequency of occurrence of uncertain events in terms of a probability density function.

\subsection{Robustness to Uncertainty}

The value of the performance level $R_{C}$ is not chosen a priori. As in all information-gap analyses, the performance level $R_{C}$ is embroiled in a basic trade-off and its value is chosen in light of the resolution of that trade-off. The basic decision function of informationgap decision theory is the robustness function $a^{*}$. The robustness of decision $q$ is the greatest value of the uncertainty parameter $a$ at which the model fidelity is 
never worse than $R_{C}$. The robustness is formally defined as:

$$
a^{*}=\underset{a \geq 0}{\operatorname{Argmax}} \max _{U\left(u_{0} ; a\right)}\left\{R(q ; u) \mid R(q ; u) \leq R_{C}\right\}
$$

The significance of the robustness function is that it assesses the degree of variation of the uncertainty $u$ that does not jeopardize the test-analysis correlation and calibration of the model. If the robustness of decision $q$ is large, then the model fidelity is immune to variations of the unknown quantities $u$. On the other hand, if $a^{*}$ is small, then even very small fluctuations of the uncertain quantities $u$ endanger the model fidelity. This implies that decisions based on the current model are likely to be questionable due to the influence of variability and modeling error.

A value of decision variables $q$ whose robustness is large represents a model from which the effect of uncertainty $u$ has been essentially removed. If $a^{*}$ is large, then we have reason to believe that $q$ represents physically meaningful properties of the system, uninfluenced by the unknown auxiliary terms $u$.

Note also that the robustness $a^{*}$ depends on the demanded model fidelity $R_{C}$. In fact, there is an irrevocable trade-off, the stricter the demanded fidelity (expressed as a small value of $R_{C}$ ), the lower the robustness (resulting in a small value of $\left.a^{*}\right) .{ }^{4}$ Good performance is obtained at the expense of eroded immunity to failure. Examining this trade-off of performance against immunity-to-failure lets the analyst choose an acceptable and feasible level of demanded fidelity $R_{C}$.

\section{IV.SHOCK PROPAgATION THROUGH A HYPER-ELASTIC MATERIAL}

The application of interest is a high-frequency shock performed in the summer of 1999 at Los Alamos National Laboratory. The experiment was designed to study the propagation of a shock wave through a nonlinear, visco-elastic material. ${ }^{5}$

\subsection{Impact Test Setup}

The test consists of dropping from various heights a carriage (drop table) on which are mounted a layer of hyper-elastic material and a steel cylinder. Upon impact on a concrete floor, a shock wave is generated that propagates through the hyper-elastic material. The heavy steel cylinder compresses the hyper-elastic pad and causes elastic and plastic strains during a few milliseconds. A photograph of the setup is shown in Figure 4-1 (a).

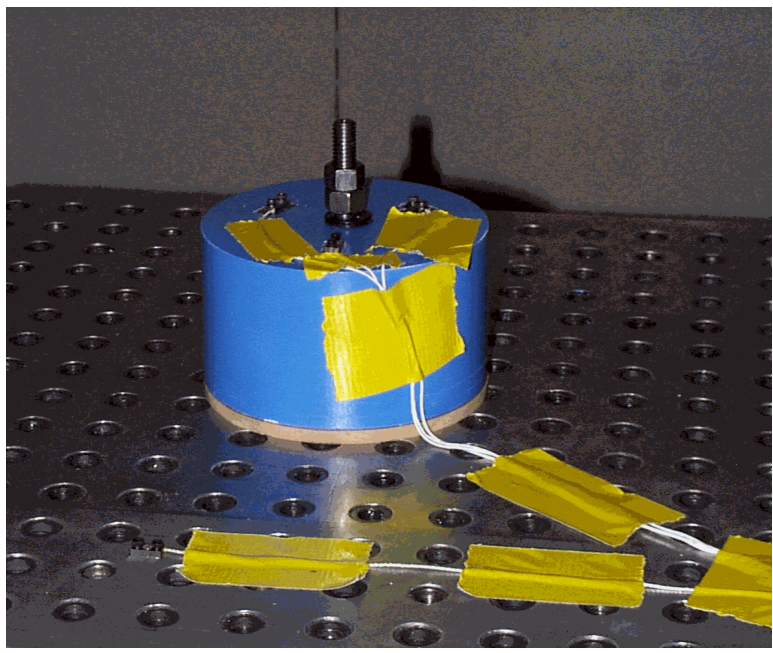

(a) Experimental set-up.

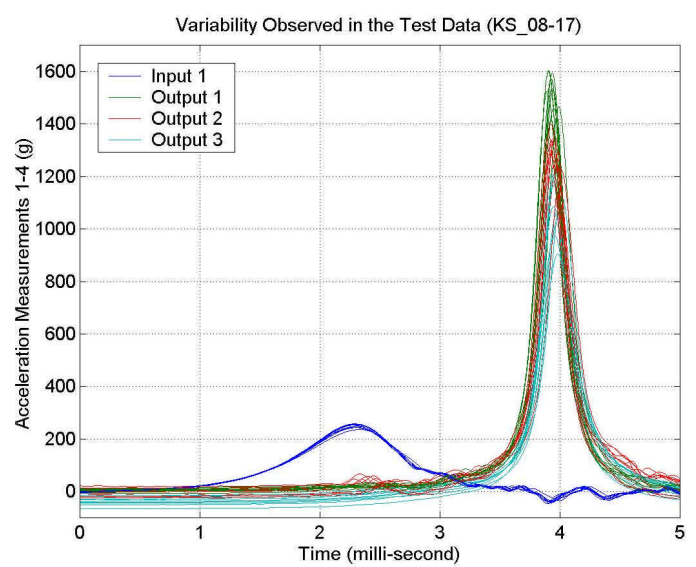

(b) Signals measured during 10 replicate tests.

Figure 4-1. Experimental set-up and acceleration measurements.

Four acceleration measurements were collected during each test. The input acceleration was measured on the top surface of the carriage and three output accelerations were measured on top of the steel cylinder. The former is referred to as the "input" acceleration signal and the latter are referred to as the "output" acceleration signals at sensors 1,2 and 3 . Figure 4-1 (b) shows the input and output signals obtained when the same test was repeated ten times.

\section{$\underline{4.2}$ Observed Variability and Feature Definition}

The reason for replicate testing was to estimate the total variability. Because the sources of environmental and operational variability were a priori unknown, the tests were repeated to estimate the total 
experimental uncertainty. Such uncertainty corresponds to the uncertainty variables denoted by $u$ in the previous discussion. Figure 4-2 illustrates the variability of output signals collected at sensor 2 when a 6.3 mm-thick hyper-foam pad is tested and the carriage is dropped from an initial height of 0.33 meters. Overall, it can be observed that peak acceleration values vary by $4.4 \%$. Although small, ignoring this variability of the peak response might result into predictions erroneous by several hundred g's, which could yield catastrophic consequences.

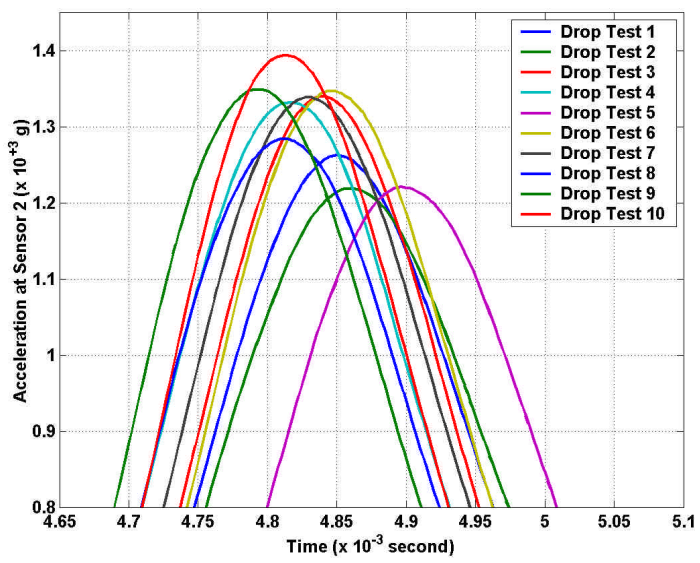

Figure 4-2. Variability measured at sensor 2.

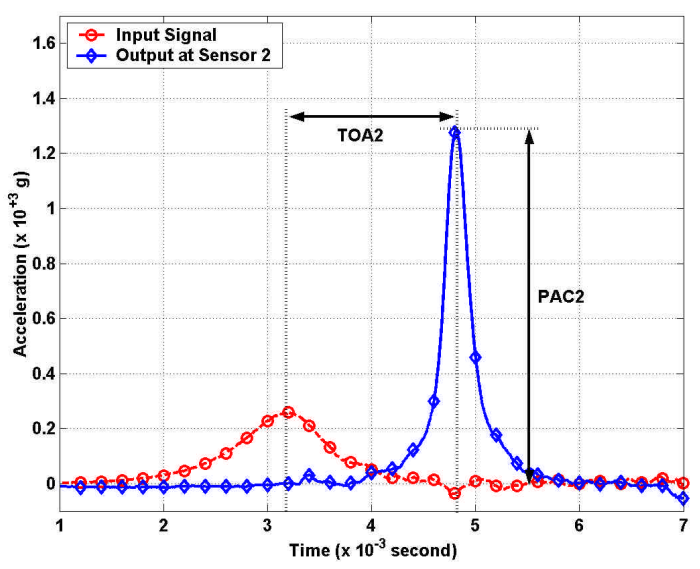

Figure 4-3. Definition of response features.

To analyze the results of this experiment, we essentially focus on the peak acceleration and time-ofarrival at sensor 2. The reason is because they are the quantities of interest to the analyst. The impulse is so short in time-and the shape of the pulse can be reproduced by a half-sine wave-that matching these two features is sufficient to capture the response's energy content. In the following, these features of the measured data are denoted by the acronyms PAC2 and

Accepted for unlimited, public release on June 11, 2002.

American Institute of Aeronautics and Astronautics.
TOA2. Figure 4-3 defines graphically the two features. It is emphasized that the time-of-arrival is not defined as the absolute time at which the signal peaks, but rather as the time it takes the shock wave to travel from the input sensor to the output sensor.

\section{FINITE ELEMENT MODELING AND TEST- ANALYSIS CORRELATION}

During the impact tests, it was noticed that the high-amplitude shocks-when the carriage was dropped from a height of four meters - had a tendency to induce significant cracking and damage to the sample of hyper-elastic material. These tests could therefore not be repeated using the same sample each time and a numerical simulation was developed to serve as a surrogate to testing.

\subsection{Finite Element Modeling}

Figure 5-1 illustrates the finite element model developed for numerical simulation. The analysis program used is $\mathrm{HKS} /$ Abaqus $^{\circledR}$-Explicit, a generalpurpose package for finite element modeling of nonlinear structural dynamics. ${ }^{6}$ It features an explicit time integration algorithm, which is convenient when dealing with non-linear material behavior, contact dynamics and high frequency excitation. The model shown in Figure 5-1 defines 963 nodes, 544 volume elements and two contact pairs located at the cylinder/pad interface and the pad/carriage interface. It yields a total of 2,889 degrees of freedom composed of structural translations in the three directions and Lagrange multipliers defined for handling the contact constraints. An analysis running on a typical singleprocessor workstation is executed in approximately 10 minutes of CPU time.

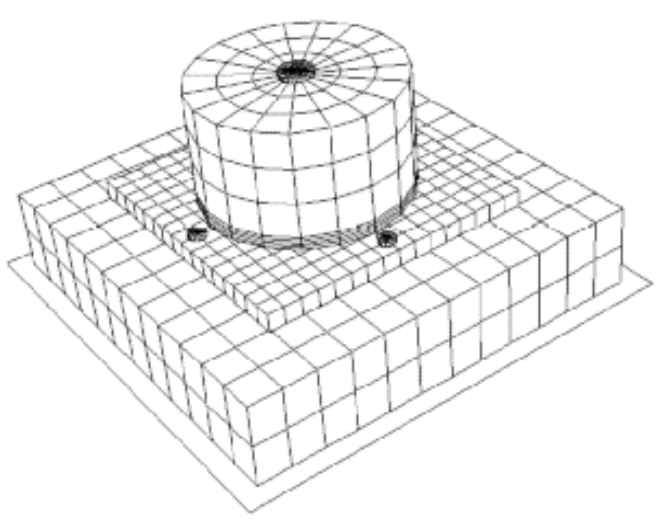

Figure 5-1. Computational model. 
The finite element simulation had to be parameterized in an effort to capture the material variability, the experimental variability and other sources of uncertainty. Based on experimental evidence, see Figure 4-1(b), it was decided that the model had to be three-dimensional to represent the fact that the drop table might not always hit the floor perfectly horizontal. Two tilt angles were therefore introduced in the numerical simulation. Another source of variability was the torque applied to the tightening bolts that held the assembly together on the carriage. The applied torque was not measured during testing. A small scaling variation of the measured impulse was also allowed to account for potential sensor calibration errors and other systematic bias introduced by data decimating and filtering. These four parameters are defined in Table 5-1. Other input parameters are not included in this analysis because previous work has demonstrated that these additional parameters do not explain the observed variability. ${ }^{5,7}$ The tilt angles, bolt preload and input scaling parameters are analogous to the decision variables $q$ :

$$
q=\left\{\begin{array}{c}
t_{1} \\
t_{2} \\
P_{B} \\
s_{I}
\end{array}\right\}
$$

Table 5-1. Input parameters of the model.

\begin{tabular}{|c|l|c|}
\hline Symbol & \multicolumn{1}{|c|}{ Definition } & Units \\
\hline$t_{l}$ & First tilt angle & Degree \\
\hline$t_{2}$ & Second tilt angle & Degree \\
\hline$P_{B}$ & Bolt preload & $\mathrm{N} / \mathrm{m}^{2}$ \\
\hline$s_{I}$ & Input magnitude scaling & Unit-less \\
\hline
\end{tabular}

Table 5-2. Ranges of input parameters.

\begin{tabular}{|c|c|c|}
\hline Symbol & Design Domain & Nominal Value \\
\hline$t_{l}$ & $0-1$ degree & 0.5 degree \\
\hline$t_{2}$ & $0-1$ degree & 0.5 degree \\
\hline$P_{B}$ & $0-3.45 \mathrm{MPa}$ & $1.72 \mathrm{MPa}$ \\
\hline$S_{I}$ & $0.9-1.1$ & 1.0 \\
\hline
\end{tabular}

Ranges for the four parameters that control the numerical simulation are shown in Table 5-2. The range of each parameter is established based on experience and physical constraints. For example, observation of the carriage's support system suggests that tilt angles greater than one degree are not possible.

\subsection{Test-analysis Correlation and Calibration}

A typical test-analysis correlation study would then proceed with a comparison of measured and predicted features to assess the model's accuracy. When the agreement is not deemed sufficient, the input parameters shown in equation (5.1) are calibrated to reduce the distance between features. This calibration concept is illustrated in Figure 5-2. It shows a " $2 \mathrm{D}$ slice" of the four-dimensional test-analysis metric $R(q)$ defined to assess the distance between measured and predicted features. The metric $R(q)$ is defined from the weighted $\mathrm{L}^{2}$ norm of the prediction error $e(q)$ :

$$
\begin{gathered}
y(q)=\left\{\begin{array}{l}
\mathrm{PAC} 2 \\
\mathrm{TOA} 2
\end{array}\right\} \\
e(q)=y^{\text {Test }}-y(q) \\
R(q)=e(q)^{T} W_{e e}^{-1} e(q)
\end{gathered}
$$

where the weighting $W_{e e}$ is, here, a constant and diagonal matrix that eliminates the difference of magnitude between units of PAC2 and TOA2.

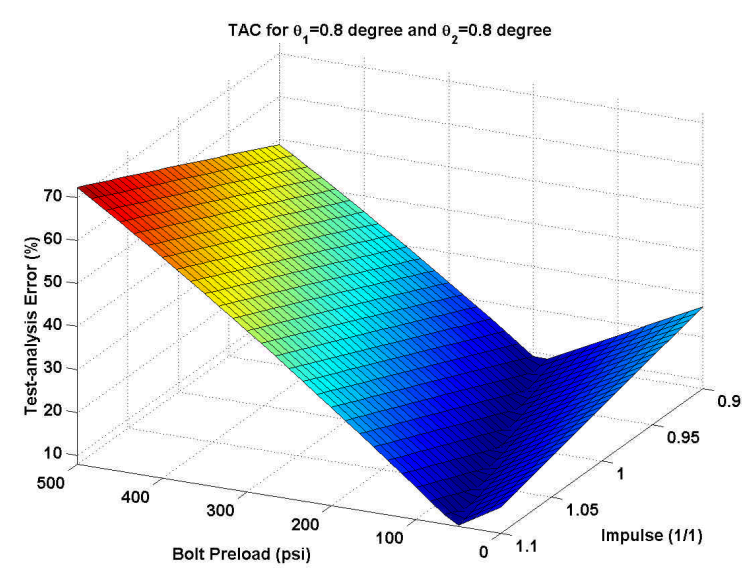

Figure 5-2. Test-analysis metric $R(q ; u)$.

Figure 5-2 shows that various combinations of preload $P_{B}$ and scaling $s_{I}$ can provide less than $10 \%$ error when both tilt angles are kept constant and equal to 0.8 degrees. The response surface was generated by analyzing the finite element model at 256 different combinations $\left(t_{1} ; t_{2} ; P_{B} ; s_{I}\right)$. Quadratic polynomials were then best fitted through the 256 features PAC2 and TOA2 to interpolate the surface between the points that had not been analyzed. In a typical optimization framework, the shape of the response surface would be unknown and an optimization solver would be wrapped around the finite element analysis code to solve the problem:

$$
q^{*}=\underset{q_{\min } \leq q \leq q_{\max }}{\operatorname{Argmin}} R(q)
$$


Hence, the ranges listed in Table 5-2 define the fourdimensional hyper-cube design domain within which parameter calibration is sought. The value of the metric at the optimal parameter set, $R^{*}=R\left(q^{*}\right)$, is then the best possible test-analysis error that can be achieved with the current model.

\subsection{Issues That Make This Approach Inadequate}

The test-analysis correlation and calibration framework of Section 5.2 breaks down in the context of uncertainty. When variability must be taken into account, the form of the uncertainty must always be assumed one way or another. Most engineering applications, for example, assume the theory of probability. Sampling techniques are then available to propagate uncertainty. Interestingly, the calibration problem (5.3) can be solved much the same way. The reader is referred to References ${ }^{8,9}$ for a discussion of uncertainty assessment, calibration, validation, and other issues. One noticeable difference is that the testanalysis correlation metric $R(q)$ should be defined in terms of a statistical test in conventional statistics ${ }^{10}$ or a posterior probability in Bayesian statistics ${ }^{11}$.

In the case of the impact test, it is known that parameters such as the tilt angles and bolt preload vary in unknown ways. Measurements are not available that would suggest a particular probability structure. Assuming that these parameters are uniformly distributed or normally distributed would be pure conjecturing at this point because evidence is simply not available to support this assumption. Making assumptions that are not backed up by strong evidence can lead to erroneous decision-making with catastrophic consequences and our claim is that analysts should be careful not to assume more than is really known".

Because there is no evidence available to suggest that probability theory is a justifiable choice to model the uncertainty sources of the impact experiment,

\footnotetext{
II See, for example, a discussion in Reference ${ }^{12}$ of the toyproblem "Can the value of $y$ be greater than 1.8 when $y=(a+b)^{a}$ and $0.1<a<1, \quad 0<b<1$ ?" Assuming Gaussian probability distributions for parameters $a, b$ and performing a one million-sample Monte Carlo analysis yields the answer "Never", which is erroneous. Assuming uniform probability distributions under-predicts the number of occurrences when $y>1.8$. If the value $y=1.8$ were some sort of critical level not to be exceeded, catastrophic failure could occur as a result of assuming more than is really known-which is that parameter $a$, likewise $b$, belongs to an interval, $0.1<a<1$. The location within the interval of the most likely value of parameter $a$ cannot be inferred from the above problem statement.
}

Accepted for unlimited, public release on June 11, 2002.

American Institute of Aeronautics and Astronautics. conventional methods for propagating uncertainty, such as statistical testing, cannot be implemented. In addition, statistical testing cannot be applied to define test-analysis correlation metrics because statistical testing generally assumes the form of the underlying distribution. It is to remedy these deficiencies that the information-gap theory of uncertainty is implemented in the remainder of this paper.

\section{ROBUSTNESS OF THE DESIGN TO UNCERTAINTY}

From now on, it is assumed that the only knowledge is that the input parameters $\left(t_{1} ; t_{2} ; P_{B} ; s_{I}\right)$ of the finite element simulation belong to the design domain defined in Table 5-2. No evidence is available from which a probability structure might be inferred and the analysts are not willing to make further assumptions.

The first question we would like to answer is "How robust is our design objective to the uncertainty in the input parameters?" Here, the objective is to ensure that the predictions of the finite element model provide an acceptable test-analysis correlation. Clearly, "acceptable" is a relative and somewhat arbitrary notion that depends on the application. For simplicity, the illustration will target an acceptance level of no more than $R_{C}=5 \%$ error. Answering the above question is, in a sense, a problematic of sensitivity. We wish to estimate the sensitivity of the design objective $R(q ; u)$ to variations of the uncertainty variables $u$.

To stress a parallel with the discussion of Section 3 , it is emphasized that input parameters $\left(t_{1} ; t_{2} ; P_{B} ; s_{I}\right)$ should from now on be labeled as uncertainty variables $u$ because the characteristics of their variations are not known with certainty. An obvious benefit of the aforementioned robustness analysis is to help us differentiate between decision variables $q$ and uncertainty variables $u$. This would typically be achieved by categorizing each input parameter either as a decision variable $q_{k}$ or an uncertain variable $u_{k}$ in an attempt to obtain a design objective $R(q ; u)$ immune to the uncertainty as much as possible.

\subsection{Numerical Procedure}

A generic robustness analysis consists of estimating the uncertainty level $a^{*}$ that provides an acceptable performance. For simplicity, the acceptable performance region is denoted by $R \leq R_{C}$ and the failure region is $R>R_{C}$. Uncertainty is represented using an information-gap model denoted by $U\left(u_{0} ; a\right)$, as outlined in Section 3. The computational and decision- 
making procedures are conceptually illustrated in Figures 6-1 (a) and (b), respectively. Figure 6-1 (a) shows that, at each uncertainty level $a_{k}$, an optimization problem must be solved that provides the worst possible performance $R^{*}\left(a_{k}\right)$ given the information-gap uncertainty bound $U\left(u_{0} ; a_{k}\right)$ :

$$
R^{*}\left(a_{k}\right)=\max _{U\left(u_{0} ; a_{k}\right)} R(q ; u)
$$

The sequence of points $\left\{a_{k} ; R^{*}\left(a_{k}\right)\right\}$ is then used to approximate the performance curve $R^{*}(a)$ shown in Figures 6-1 (a) and (b). Information therefore flows from the vertical axis $(a)$ to the horizontal axis $\left(R^{*}\right)$. Decision-making is illustrated in Figure 6-1 (b) and it reverses the flow of information. For the target performance $R_{C}$, the allowable uncertainty $a^{*}$ is obtained by reading the performance curve $R^{*}(a)$. The shaded area in Figure 6-1 (b) hence represents the acceptable operating region.

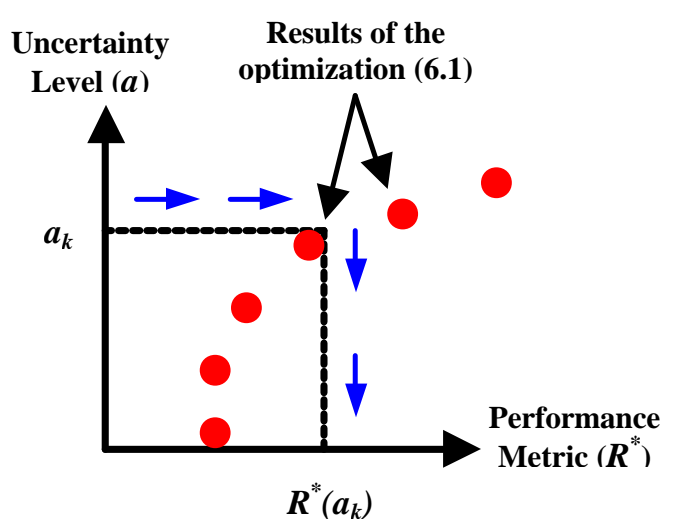

(a) Info-gap robustness calculation.

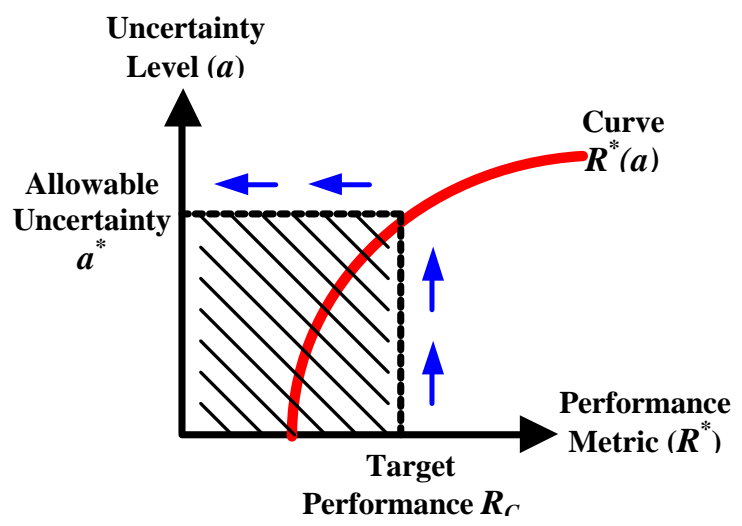

(b) Inference of the allowable uncertainty.

Figure 6-1. Conceptual info-gap analysis.

Of course, if the analyst is only interested in estimating $a^{*}$, it is not necessary to reconstruct the

Accepted for unlimited, public release on June 11, 2002. American Institute of Aeronautics and Astronautics. entire curve $R^{*}(a)$ and efficient search strategies can be devised that take advantage of its proven monotony. ${ }^{13}$

\subsection{Robustness of the Impact Experiment}

The information-gap robustness analysis is summarized in Figure 6-2 for the impact experiment. The objective is to assess the robustness of testanalysis correlation to input parameter uncertainty. Therefore, the adverse aspect of uncertainty is investigated by answering the question: "By how much does uncertainty deteriorate the original correlation?"

The analysis starts with the definition of four information-gap uncertainty levels $a_{1}-a_{4}$, one for each design parameter. The robustness analysis focuses on one uncertain variable $u_{k}$ at a time, with the other three kept constant and equal to their nominal value. This approach lets us study the effect of each input parameter independently from the others. The performance criterion $R(q ; u)$ is the test-analysis correlation metric defined in equation (5.2) and the convex domains of uncertainty $U\left(u_{0} ; a\right)$ are defined by:

$$
\begin{gathered}
u_{B}=\left(u_{\text {Max }}-u_{\text {Min }}\right) / 2 \\
\left(u_{0}-a u_{B}\right) \leq u \leq\left(u_{0}+a u_{B}\right)
\end{gathered}
$$

where $u_{\text {Min }}, u_{0}$ and $u_{\text {Max }}$ are the minimum, nominal and maximum values of each variable defined in Table 5-2. For any given info-gap $a$, the uncertain variable $u$ can vary in the interval from $\left(u_{0}-u_{B}\right)$ to $\left(u_{0}+u_{B}\right)$. However, because the horizon-of-uncertainty parameter $a$ is in fact not known, we confront here not a single specific interval of variation $U\left(u_{0} ; a\right)$, but rather a family of nested uncertainty intervals $\left\{U\left(u_{0} ; a\right), a>0\right\}$.

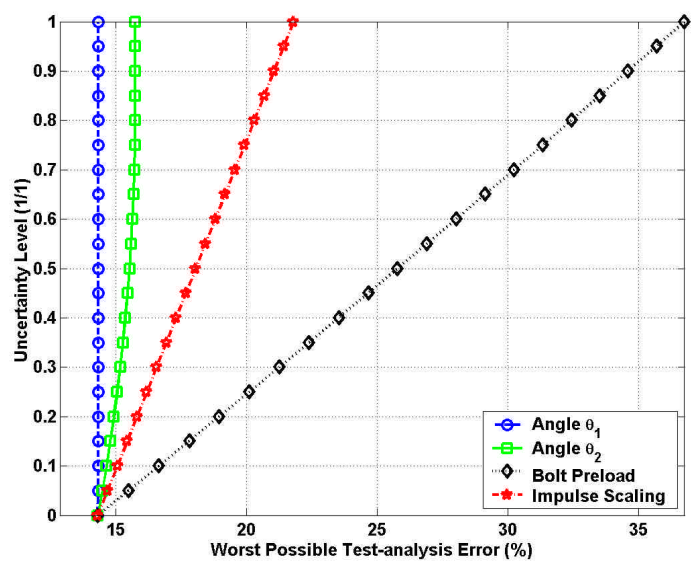

Figure 6-2. Worst-case info-gap robustness.

Figure 6-2 investigates the deterioration of the original test-analysis correlation due to uncertainty. 
The optimization searches for the worst correlation at each uncertainty level. A gradient-based BFGS algorithm is wrapped around the HKS/Abaqus ${ }^{\circledR}$ finite element package through a Matlab ${ }^{\mathrm{TM}}$ interface. This implementation is reasonable only because the algorithm optimizes one variable at a time and requires a small number of finite element calculations to converge, typically 12 to 15 . It can be observed that the most adverse effects of uncertainty are associated with parameters $P_{B}$ and $s_{I}$. Uncertainty in the preload $P_{B}$ has the potential to increase the original $14.5 \%$ correlation error to more than $37 \%$. Uncertainty in the tilt angles, on the other hand, produces little deterioration of the correlation. From this analysis, we learn that it may not be critical during an experiment to attempt to control or measure the tilt angles.

\subsection{Opportunity From Uncertainty}

We now investigate the beneficial aspect of uncertainty. The question is: "Can uncertainty be taken advantage of to improve the test-analysis correlation?"

This question is not to be confused with the previous analysis of robustness. It is in fact defined in Reference $^{3}$ as a judgment of opportunity. Robustness searches for the greatest info-gap parameter $a^{*}$ at which $R(q ; u)$ is necessarily less than the performance level $R_{C}$. It results in two embedded maximization problems, as shown in equation (3.4). Opportunity, on the other hand, is the least info-gap parameter at which $R(q ; u)$ can be, but is not necessarily, less than $R_{W}$. To stress the difference with the robustness function previously denoted by $a^{*}$, the opportunity is denoted by $b^{*}$ :

$$
b^{*}=\underset{a \geq 0}{\operatorname{Argmin}} \min _{U\left(u_{0} ; a\right)}\left\{R(q ; u) \mid R(q ; u) \leq R_{W}\right\}
$$

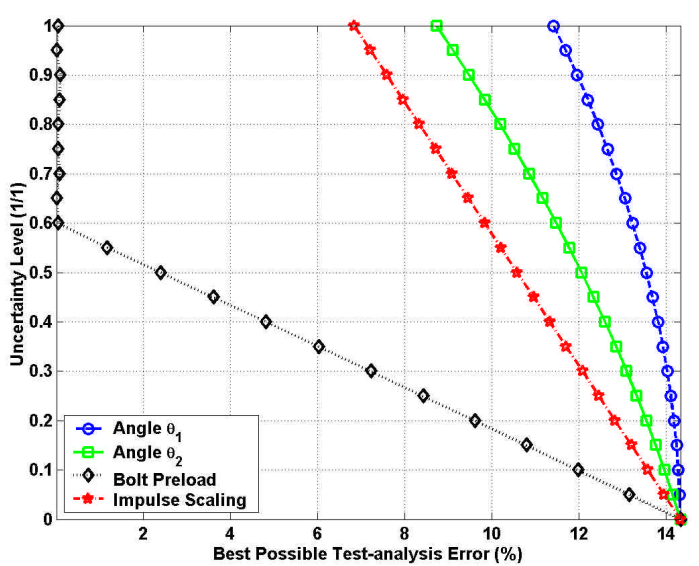

Figure 6-3. Best-case info-gap opportunity.

Accepted for unlimited, public release on June 11, 2002.

American Institute of Aeronautics and Astronautics.
The threshold $R_{W}$ is the windfall performance and it is generally desirable to choose it much smaller than the critical performance $R_{C}, R_{W}<<R_{C}$. In our application, for example, the critical performance is the test-analysis correlation error above which the model's predictions are deemed inappropriate, $R_{C}=5 \%$. The windfall performance $R_{W}$ defines what would essentially be a "perfect" model with, say, less than $R_{W}=0.1 \%$ test-analysis correlation error.

Figure 6-3 investigates the beneficial effects of uncertainty. The optimization searches for the best possible correlation at each uncertainty level. Clearly, letting all four parameters venture away from their nominal settings has the potential of reducing significantly the correlation error. This is especially dramatic with the preload $P_{B}$. From this analysis, we learn that the target $R_{W}=0.1 \%$ error can be met by letting the preload parameter vary. It does not, however, mean that a model $q$ that yields "perfect" predictions will necessarily be obtained when $b^{*}=60 \%$ uncertainty is tolerated in the preload. It just means that it is a possibility that could happen.

\subsection{How Good is Good Enough?}

It must also be recognized that an acceptance level such as $R_{C}$ or $R_{W}$ is always somewhat arbitrary. For example, NASA standards call for a 5\% maximum error between test and analysis modal frequencies. ${ }^{14}$ The corresponding Air Force standard is 2\%. A legitimate question is to ask the extent to which a model that meets the NASA standard is unacceptable by Air Force standard. Conversely, is 7\% error truly much worse than $5 \%$ ? This problematic is captured by the simple question "How good is good enough?" and the info-gap robustness and opportunity analyses can help decision-makers answer this difficult question.

The reason is because information-gap robustness and opportunity not only assess the worst and best potential performances of the system in the presence of uncertainty but also permit to identify their trends as the level of uncertainty increases or decreases.

Consider, for example, Figure 6-3 where it can be observed that tolerating $40 \%$ uncertainty in the knowledge of the bolt preload $P_{B}$ could provide a decision model $q$ that meets the performance criterion of $5 \%$ error. The curve also shows that allowing $60 \%$ uncertainty could potentially yield a perfect prediction with less than $0.01 \%$ error. If an analyst is considering a validation experiment to test the design $40 \%$ away from the nominal preload setting, the potential benefits of allowing a little more uncertainty $-60 \%$ instead of $40 \%$-are now clear. This simple example illustrates 
the potential of information-gap analysis for costbenefit decision.

\section{CALIBRATION UNDER UNCERTAINTY}

After having studied the effect of each uncertainty variable $u_{k}$ on the performance criterion $R(q ; u)$, the robustness problem is now solved. The objective is to identify the allowable amount of uncertainty $a^{*}$ that provides an acceptable design. The four variables $t_{1}, t_{2}, P_{B}$ and $s_{I}$ are investigated jointly even though the analysis could be restricted to $P_{B}$ and $s_{I}$ because it has been demonstrated in Section 6.2 that the prediction error is robust to tilt angle uncertainty.

Following the procedure described in Section 6.1, an optimization problem is solved to calculate the uncertainty level for which the model prediction does not exceed the $R_{C}=5 \%$ error:

$$
a^{*}=\underset{0 \leq a \leq 1}{\operatorname{Argmax}} \max _{\left(u_{0} ; a\right)}\left\{R(q ; u) \mid R(q ; u) \leq R_{C}\right\}
$$

The convex domain of uncertainty $\left\{U\left(u_{0} ; a\right), a>0\right\}$ is the family of nested four-dimensional ellipsoids whose dilatation in each direction is controlled by a diagonal weighting $W_{u и}$ :

$$
U\left(u_{0} ; a\right)=\left\{u \mid\left(u-u_{0}\right)^{T} W_{u u}\left(u-u_{0}\right) \leq a\right\}, a>0
$$

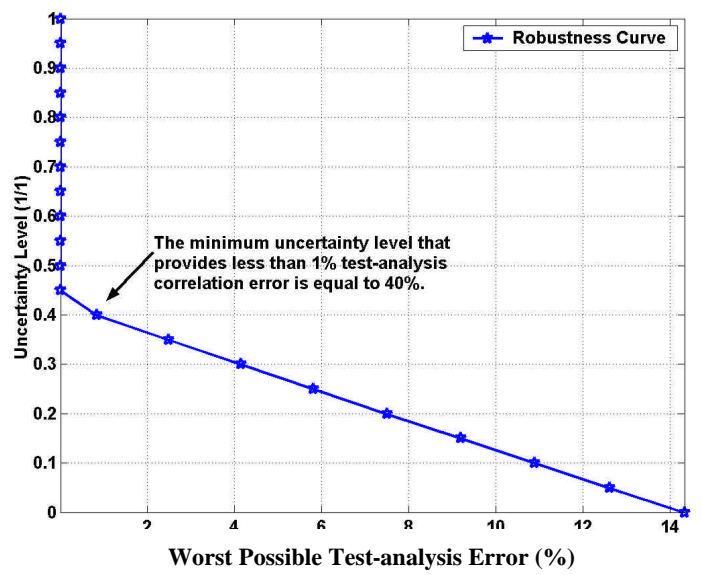

Figure 7-1. Parametric info-gap calibration.

Figure 7-1 shows the robustness curve $a$ versus $R^{*}(a)$, from which the optimal solution $a^{*}$ of the problem (7.1) can be read directly. A total uncertainty level of $28 \%$ provides less than $5 \%$ test-analysis correlation error while the uncertainty level must be increased to $40 \%$ to reach less than $1 \%$ error. The variables $\left(t_{1} ; t_{2} ; P_{B} ; s_{I}\right)$ that yield less than $1 \%$ error are listed in Table 7-1.

Table 7-1. Design that provides $1 \%$ error.

\begin{tabular}{|c|c|c|}
\hline Symbol & Nominal Value & Calibrated Value \\
\hline$t_{l}$ & 0.50 degree & 0.97 degree \\
\hline$t_{2}$ & 0.50 degree & 0.98 degree \\
\hline$P_{B}$ & $1.72 \mathrm{MPa}$ & $1.28 \mathrm{MPa}$ \\
\hline$s_{I}$ & $100.00 \%$ & $92.52 \%$ \\
\hline
\end{tabular}

The example demonstrates that the input parameters can be calibrated under uncertainty to improve the model's predictive accuracy. Beyond calibration, valuable information is learned about the overall level of uncertainty that affects the system's performance. The $28 \%$ uncertainty level may be, for example, translated into a specification of the amount of torque applied to tighten the bolts. The value of this analysis is therefore to provide a quantitative assessment of the relationship between uncertainty and performance, without having to rely on probabilistic or approximation methods.

\section{HYBRID UNCERTAINTY MODELS}

Our final application investigates the possibility of nesting information-gap models within each other to explore not only the design space but also the form of the model itself. So far, we have only considered a single IGM $U\left(u_{0} ; a\right)$ and a single uncertainty parameter $a$ associated with the variability of parameters $u$. The concept is now generalized to define a second IGM $V\left(v_{0} ; c\right)$ that represents a particular family of models. The associated uncertainty parameter is denoted by $c$ and the variables that describe the space of potential model forms are denoted by $v$. The two models are embedded, that is, a given value of $v$ provides a particular model form within which the parameters $u$ can exhibit variations, as before.

It is assumed that we wish to characterize the variability of parameters $u$ through a matrix $W_{v v}$ of covariance coefficients:

$$
W_{v v}=E\left[\left(u-u_{0}\right)\left(u-u_{0}\right)^{T}\right]
$$

Reasons might be that another analysis code has to be used that can only handle randomness through the definition of probability density functions. The preferred scientific method would be to estimate the covariance matrix $W_{v v}$ from measurements of $u$ in replicate experiments. We have seen that $u$ can however not be measured directly. 
The inference procedure is therefore started by defining the IGM $V\left(v_{0} ; c\right)$. The parameter $v$ symbolizes the covariance coefficients of the statistical model of the uncertainty variables $u$. Parameter $c$ represents the info-gap uncertainty associated with $v$. In statistical sciences, the parameters $v$ are sometimes referred to as the "hyper-parameters" because they represent the unknown statistics of variables $u$. Lack of direct evidence prohibits the assumption of a probability structure for the hyper-parameters $v$ but it is nevertheless assumed that the four variables $u$ are uncorrelated with the exception of the two tilt angles":

$$
W_{v v}=\left[\begin{array}{cccc}
v_{1} & v_{5} & 0 & 0 \\
v_{5} & v_{2} & 0 & 0 \\
0 & 0 & v_{3} & 0 \\
0 & 0 & 0 & v_{4}
\end{array}\right]
$$

The original problem of determining the influence of four variables $u$ is therefore augmented with the four coefficients of variance $v_{1}-v_{4}$ and the covariance coefficient $v_{5}$ between tilt angles $t_{1}$ and $t_{2}$. Table 8-1 defines the nominal variance and covariance coefficients and their assumed ranges. The resulting model $U\left(u_{0} ; a\right) x V\left(v_{0} ; c\right)$ defines a hybrid model of uncertainty where the probabilistic and info-gap theories of uncertainty are embedded. ${ }^{3}$

Table 8-1. Ranges of hyper-parameters.

\begin{tabular}{|c|c|c|}
\hline Symbol & Design Domain & Nominal Value $^{2}$ \\
\hline$v_{l}$ & $0-15.0$ degree $^{2}$ & 0.0 degree $^{2}$ \\
\hline$v_{2}$ & $0-15.0$ degree $^{2}$ & 0.0 degree $^{2}$ \\
\hline$v_{3}$ & $0-5,160.0 \mathrm{Mpa}^{2}$ & $0.0 \mathrm{MPa}^{2}$ \\
\hline$v_{4}$ & $0-16.5$ & 0.0 \\
\hline$v_{5}$ & $0-15.0$ degree & 2 \\
\hline
\end{tabular}

(Variance and covariance coefficients, $\times 10^{-3}$.)

The performance criterion $R(q ; u)$ needs to be adapted to the new information-gap models $U\left(u_{0} ; a\right)$ and $V\left(v_{0} ; c\right)$. It translates into a test-analysis metric $R(q ; u ; v)$ that is analogous to a posterior probability in the sense of Bayesian statistics:

$$
\begin{gathered}
e(q ; u)=y^{\text {Test }}-y(q ; u) \\
\delta q=q-q_{0} \\
R(q ; u ; v)=e(q ; u)^{T} W_{e e}^{-1} e(q ; u)+\delta q^{T} W_{v v}^{-1} \delta q
\end{gathered}
$$

\footnotetext{
\# The reason for this choice is that there is no physical reason why the parameters $P_{B}$ and $s_{I}$ should be correlated with each other and the tilt angles. The carriage's support system, on the other hand, might introduce a correlation between the tilt angles $t_{1}$ and $t_{2}$.
}

Accepted for unlimited, public release on June 11, 2002. American Institute of Aeronautics and Astronautics. where $W_{v v}$ is the covariance matrix defined in equation (8.2) and $W_{e e}$ now denotes the covariance matrix of the PAC2 and TOA2 features, estimated from the ten replicate experiments:

$$
W_{e e}=\left[\begin{array}{cc}
3.46 \times 10^{+3} & -1.34 \times 10^{-3} \\
-1.34 \times 10^{-3} & 9.61 \times 10^{-10}
\end{array}\right]
$$

Once the IGM $U\left(u_{0} ; a\right)$ and $V\left(v_{0} ; c\right)$ and the performance function $R(q ; u ; v)$ have been defined, the numerical implementation is similar to the one described in Section 6.1. Essentially, the optimal uncertainty levels $a^{*}, c^{*}$ and decision variables $q^{*}$ are sought that satisfy the criterion $R(q ; u ; v) \leq 5 \%$.

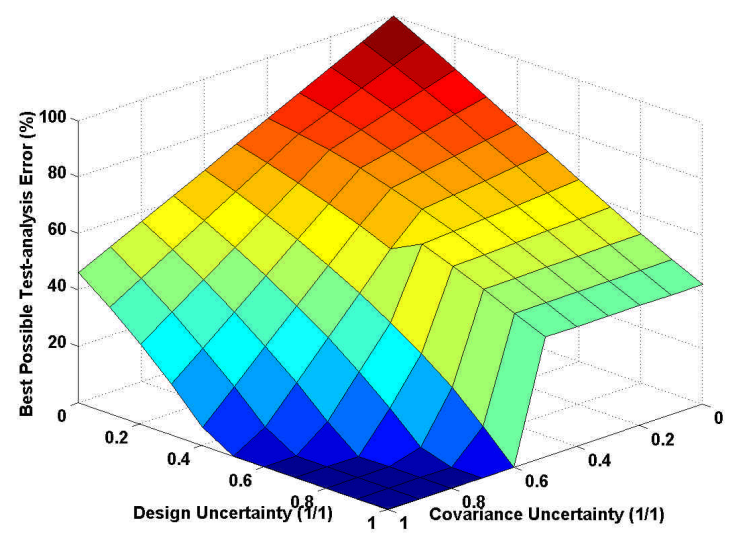

Figure 8-1. Calibration of the hybrid model.

Figure 8-1 illustrates the performance metric $R(q ; u ; v)$ versus uncertainty levels $a$ and $c$. Each point on the surface represents the result of the optimization of variables $(u ; v)$ in a specific convex domain $U\left(u_{0} ; a\right) \times V\left(v_{0} ; c\right)$. Because the number of unknowns is small (four variables $u$ plus five coefficients of variance $v$ ), convergence is generally reached with 15 to 20 finite element analyses. The region of the design space that provides less than 5\% error is clearly visible. Covariance structure uncertainty and design parameter variability exhibit a complex pattern of interaction. With small covariance coefficients (small $c$ ), the performance is essentially governed by the variables $u$. As the covariance coefficients become more pronounced and the parametric variability of $u$ increases, a dramatic change occurs. The interaction between the two sources of uncertainty suddenly yields much better performance criteria $R(q ; u ; v)$.

Calibration results are summarized in Table 8-2 for the variables $u$ and Table 8-3 for the coefficients $v$ of variance and covariance. The resulting standard deviations of $t_{1}, t_{2}, P_{B}$ and $s_{I}$ are listed in Table 8-4 and 
the correlation coefficient between tilt angles $t_{1}$ and $t_{2}$ is equal to $26.5 \%$.

Table 8-2. Design that provides $1 \%$ error.

\begin{tabular}{|c|c|c|}
\hline Symbol & Nominal Value & Calibrated Value \\
\hline$t_{I}$ & 0.50 degree & 0.99 degree \\
\hline$t_{2}$ & 0.50 degree & 0.29 degree \\
\hline$P_{B}$ & $1.72 \mathrm{MPa}$ & $2.61 \mathrm{MPa}$ \\
\hline$s_{I}$ & $100.00 \%$ & $103.01 \%$ \\
\hline
\end{tabular}

Table 8-3. Calibrated hyper-parameters.

\begin{tabular}{|c|r|r|r|r|}
\hline Symbol & \multicolumn{1}{|c|}{$t_{1}$} & \multicolumn{1}{|c|}{$t_{2}$} & \multicolumn{1}{|c|}{$P_{B}$} & \multicolumn{1}{|c|}{$s_{I}$} \\
\hline$t_{1}$ & 4.8 & 1.5 & 0 & 0 \\
\hline$t_{2}$ & 1.5 & 6.7 & 0 & 0 \\
\hline$P_{B}$ & 0 & 0 & $3,741.5$ & 0 \\
\hline$s_{I}$ & 0 & 0 & 0 & 11.8 \\
\hline
\end{tabular}

(Variance and covariance coefficients, $\mathrm{x} 10^{-3}$.)

Table 8-4. Inferred standard deviations.

\begin{tabular}{|c|c|c|}
\hline Symbol & Standard Deviation & \% of Mean \\
\hline$t_{l}$ & 0.07 degree & $7.0 \%$ \\
\hline$t_{2}$ & 0.08 degree & $28.3 \%$ \\
\hline$P_{B}$ & $1.93 \mathrm{MPa}$ & $74.1 \%$ \\
\hline$s_{I}$ & 0.11 & $0.1 \%$ \\
\hline
\end{tabular}

The first conclusion that can be drawn from this analysis is that, although the correlation between the tilt angles is relatively small at $26.5 \%$, it seems important that it is included in the model to obtain a good correlation with test results. Such information would be valuable to proceed with the development of a probabilistic uncertainty model.

Comparing the calibration results from Tables 7-1 and 8-2 shows that the calibrated values of the first angle $t_{l}$ and the input magnitude scaling $s_{I}$ are very consistent. This is confirmed by the less-than-10\% standard deviations for $t_{I}$ and $s_{I}$ shown in Table 8-4. Similarly, the significant standard deviations for $t_{2}$ and $P_{B}$ are confirmed by the differences of calibrated values read from Tables 7-1 and 8-2 for the design variables $t_{2}$ and $P_{B}$.

The example illustrates the potential of info-gap analysis to learn valuable information about the uncertainty pattern of a non-linear structural dynamics experiment, without having to assume any particular probability structure. The information provided by

\footnotetext{
** The standard deviation coefficients listed in Table 8-4 are equal to the square roots of variance coefficients shown on the main diagonal of Table 8-3. The covariance coefficient between angles $t_{1}$ and $t_{2}$ is defined as the angle between column 1 (for $t_{1}$ ) and column 2 (for $t_{2}$ ) of Table 8-3.
}

Figure 8-1 is also quite important in the context of cost-benefit analyses to trade-off experimentation costs with the consequences of not testing certain regions of the design or operational space.

\section{CONCLUSION}

The quantification and propagation of uncertainty through a linear model has been the subject of extensive studies in many sciences, especially in the context of probability theory. The relationship between uncertainty and non-linear dynamics is not so well defined, the main reason being the difficulty to analyze non-linear systems.

This paper illustrates how information-gap models can be defined and analyzed to propagate uncertainty through a non-linear finite element simulation without relying on probabilities. The nonlinear system considered is the propagation of a transient impact through a layer of hyper-elastic material. The sources of non-linearity are the softening of the constitutive law of the hyper-elastic material and contact dynamics at the interface between metallic and crushable materials.

Several problems commonly encountered in structural dynamics-robustness of the design performance to uncertainty, calibration under uncertainty, statistical inference - are illustrated in the context of test-analysis correlation. This work demonstrates that such problems can be formulated and solved without ever having to rely on the theory of probability. Analysts are therefore offered with a practical alternative in situations where sparse data are available or limited testing is possible. The main limitation of the approach remains in its computational burden because a sequence of numerical optimization problems must be solved to assess the effect of uncertainty on a performance criterion.

Future work will focus on the application of information-gap to decision-making and model validation. Model validation-or the assessment of a numerical model's predictive accuracy in a design or operational space-requires correlation with test data. In the case of complex engineering systems, it is doubtful, however, that enough validation experiments will ever be performed to collect enough test data sets and develop statistical models of validation. Information-gap models of validation are therefore being envisioned as, likely, the only currently available alternative. 


\section{REFERENCES}

${ }^{1}$ Saltelli, A., Chan, K., Scott, E.M., Sensitivity Analysis, Wiley Series in Probability and Statistics, Wiley and Sons, West Sussex, England, 2000.

${ }^{2}$ Mottershead, J.E., Friswell, M.I., "Model Updating in Structural Dynamics: A Survey," Journal of Sound and Vibration, Vol. 162, No. 2, 1993, pp. 347-375.

${ }^{3}$ Ben-Haim, Y., Information-Gap Decision Theory: Decisions Under Severe Uncertainty, Series on Decision and Risk, Academic Press, 2001.

${ }^{4}$ Ben-Haim, Y., "Robust Rationality and Decisions Under Severe Uncertainty," Journal of the Franklin Institute, Vol. 337, 2000, pp. 171-199.

${ }^{5}$ Schultze, J.F., Hemez, F.M., Doebling, S.W., Sohn, H., "Statistical Based Non-linear Model Updating Using Feature Extraction," $19^{\text {th }}$ International Modal Analysis Conference, Kissimmee, FL, Feb. 5-8, 2001, pp. 18-26.

${ }^{6}$ Abaqus ${ }^{\mathrm{TM}}$ /Explicit, User's Manual, Version 6.2, Hibbitt, Karlsson \& Sorensen, Pawtucket, RI, 2001.

${ }^{7}$ Hemez, F.M., Wilson, A.C., Doebling, S.W., "Design of Computer Experiments for Improving an Impact Test Simulation," $19^{\text {th }}$ International Modal Analysis Conference, Kissimmee, FL, Feb. 5-8, 2001, pp. 977-985.

${ }^{8}$ Doebling, S.W., Hemez, F.M., Schultze, J.F., "Validation of the Transient Structural Response of a Threaded Assembly," $4^{\text {th }}$ AIAA Non-deterministic Approaches Forum, Paper \# AIAA-2002-1644, Denver, CO, April 22-25, 2002.
${ }^{9}$ Doebling, S.W., "Structural Dynamics Model Validation: Pushing the Envelope," International Conference on Structural Dynamics Modeling: Test, Analysis, Correlation and Validation, Madeira Island, Portugal, June 3-5, 2002, to appear.

${ }^{10}$ Hemez, F.M., Doebling, S.W., "Inversion of Structural Dynamics Simulations: State-of-the-art and Orientations of the Research," $25^{\text {th }}$ ISMA International Conference on Noise and Vibration Engineering, Leuven, Belgium, Sept. 13-15, 2000, pp. 403-413.

${ }^{11}$ Hemez, F.M., Doebling, S.W., "A Validation of Bayesian Finite Element Model Updating for Linear Dynamics," $17^{\text {th }}$ International Modal Analysis Conference, Kissimmee, FL, Feb. 8-11, 1999, pp. 1545-1555.

${ }^{12}$ Oberkampf, W.L., Helton, J.C., Joslyn, C.A., Wojtkiewicz, S.F., Ferson, S., "Challenge Problems: Uncertainty in System Response Given Uncertain Parameters," Workshop of the Sandia Epistemic Uncertainty Project, Sandia National Laboratories, Albuquerque, NM, August 6-7, 2002, to appear. http://www.sandia.gov/epistemic/.

${ }^{13}$ Vinot, P. Cogan, S. Ben-Haim, Y., "Reliability of Structural Dynamics Models Based on Info-gap Models," $20^{\text {th }}$ International Modal Analysis Conference, Los Angeles, CA, Feb. 4-7, 2002, pp. 1057-1063.

${ }^{14}$ Hasselman, T.K., Coppolino, R.N., Zimmerman, D.C., "Criteria for Modeling Accuracy: A State-of-the-Practice Survey," $18^{\text {th }}$ International Modal Analysis Conference, San Antonio, TX, Feb. 7-10, 2000, pp. 335-341. 